\title{
Raman spectroscopy on amorphous carbon films
}

\author{
J. Schwan, S. Ulrich, V. Batori, and H. Ehrhardt \\ Universität Kaiserslautern, FB Physik, Schrödingerstr, 67663 Kaiserslautern, Germany \\ S. R. P. Silva \\ University of Surrey, Electronic and Electrical Engineering, Guildford, Surrey GU2 5XH, England
}

(Received 5 December 1995; accepted for publication 25 March 1996)

\begin{abstract}
The origin and interpretation of the Raman features of amorphous (hydrogenated) carbon films deposited at room temperature in the region of $1000-1700 \mathrm{~cm}^{-1}$ is discussed in this paper. Possible interpretations of the linewidths, positions of the " $G$ " graphite peak and " $D$ "' disordered peak, and their intensity ratios are examined using results obtained from magnetron sputtered and magnetic field enhanced plasma deposited films. It is shown that even small "clusters" of condensed benzene rings (cluster size below $20 \AA$ ) in carbon films can explain the observed Raman scattering. Besides the care that should be taken in the correct interpretation of Raman results, the utility of Raman scattering in obtaining an estimate of cluster sizes in amorphous (hydrogenated) carbon films is discussed. Carbon films prepared by magnetron sputtering show two additional Raman features at 1180 and $1490 \mathrm{~cm}^{-1}$ in addition to the $G$ and $D$ peaks. It is shown that a correlation exists between the $1180 \mathrm{~cm}^{-1}$ peak and the $s p^{3}$ content in the films. (C) 1996 American Institute of Physics.
\end{abstract} [S0021-8979(96)05013-X]

\section{INTRODUCTION}

Amorphous carbon and hydrogenated amorphous carbon films ( $a-\mathrm{C}$ and $a-\mathrm{C}: \mathrm{H})$ have received much scientific interest since the early 1970 s due to their useful mechanical properties. ${ }^{1-5}$ Raman spectroscopy is a nondestructive technique which is used to characterize diamondlike carbon (DLC) or polymerlike carbon films. Tamor and Vassel $^{6}$ found Raman "fingerprinting" to reliably predict hydrogenation, optical gap, and other mechanical properties. Yet, there have been no publications that can theoretically or analytically explain the observed Raman effects in $a$-C and $a-\mathrm{C}: \mathrm{H}$ thin films.

Tuinstra and Koenig ${ }^{7}$ obtained Raman spectra of singlecrystal and microcrystalline graphite samples in 1970. For single-crystal graphite they found a single line at $1575 \mathrm{~cm}^{-1}$ which was attributed to the Raman active $E_{2 g_{2}}$ mode of graphite (additionally there exists an interplanar "rigid-layer shear" mode $E_{2 g_{1}}$ at $\left.42 \mathrm{~cm}^{-1}\right)$. ${ }^{8,9}$ The $E_{2 g_{2}}$ mode is commonly referred to as the $G$ peak ( $G$ for graphite). For microcrystalline graphite, Tuinstra and Koenig found two lines centered at 1575 and $1355 \mathrm{~cm}^{-1}$. Tuinstra and Koenig assigned the $1355 \mathrm{~cm}^{-1}$ line to first-order scattering from a zone boundary phonon activated by disorder due to the finite crystallite size. Nemanich and Solin ${ }^{8}$ explained the 1355 $\mathrm{cm}^{-1}$ peak by the flatness of the phonon dispersion curves at the zone boundary (from $K$ to $M$ point). Thus, a high phonon density of states will be created, which results in peaks at $1340 \mathrm{~cm}^{-1}$ and a peak at $1470 \mathrm{~cm}^{-1}$. In literature the 1355 $\mathrm{cm}^{-1}$ mode is referred to as the $D$ peak ( $D$ for disordered).

The experiments of Tuinstra and Koenig ${ }^{7}$ were supported by calculations of Lespade, Al-Jishi, and Dresselhaus ${ }^{10}$ who made computations for Raman scattering from incompletely graphitized carbons and amorphous carbons. Lespade, Al-Jishi, and Dresselhaus ${ }^{10}$ also found that the Raman features were strongly correlated to the mean diamagnetic susceptibility, which itself is sensitive to the size of ordered two-dimensional regions in the graphitic layers. Wada and Solin ${ }^{11}$ showed that the $E_{2 g_{2}}$ mode of graphite has a 50 times higher Raman scattering cross section than the "diamond band" at $1332 \mathrm{~cm}^{-1}$. Using the results of Wada and Solin ${ }^{11}$ Nemanich et al. ${ }^{12}$ discussed Raman scattering of DLC thin films based on a carbon composite model. They concluded that the Raman scattering of DLC is similar to microcrystalline graphite from their measurements. Shroder, Nemanich, and Glass ${ }^{13}$ showed that the size of such "graphitic" regions should be islands less than 15-20 ̊. They showed that the Raman scattering in the DLC films associated with $s p^{2}$ bonding is much stronger than for crystalline or microcrystalline graphite.

Wada, Gaczi, and Solin ${ }^{14}$ in a study of $a$-C films using electron energy loss spectroscopy (EELS), electron spin (paramagnetic) resonance (ESR or EPR), and Raman spectroscopy showed their films to have planar graphitic clusters of $20 \AA$ size. Beeman et al. ${ }^{15}$ stated that the model of Wada, Gaczi, and Solin ${ }^{14}$ could be improved by incorporating fourfold coordinated carbon in the cluster matrix which allows changes in the orientation of the graphitic planes. They showed the strong influence of bond angle disorder on the phonon density of states and Raman intensity. Robertson and $\mathrm{O}^{\prime}$ Reilly ${ }^{16}$ stated that the $s p^{2}$ graphitic clusters should be terminated by $s p^{3}$ sites. Dillon, Wodam, and Katkanant ${ }^{17}$ proposed that the Raman line positions yield further information concerning the bond angle disorder and the bonding in crystallites. They also attributed the observed decrease in linewidth for the $D$ and $G$ peaks of the $a$-C films to the removal of bond angle disorder and the increasing dominance of crystallites.

$\mathrm{Li}$ and Lannin ${ }^{18}$ found in-plane correlation lengths of 15-30 $\AA$ in nanocrystalline "glassy" carbon and demonstrated that the Raman spectra represents a weighted phonon 
density of states (phonon DOS) of low frequency order by comparison with inelastic neutron scattering data. Raman scattering proved to be most useful in the characterization of carbon films when the $G$ peak and $D$ peak are distinct in the spectrum. If the carbon system is highly disordered, strong contributions to the $G$ and $D$ peak from the phonon DOS makes analysis more complicated and demanding.

We report that the observed Raman results of amorphous (hydrogenated) carbon films may be interpreted in terms of an amorphous carbon matrix of threefold and fourfold coordinated carbon atoms in which small regions of a few condensed benzene rings (after Ehrhardt et al. ${ }^{19}$ and Kleber et $a l . .^{20}$ up to five fused benzene rings) are embedded. In this article, it is shown that the so-called $D$ peak is present in condensed benzene regions even as small as one benzene ring. Thus the nomenclature "disordered" peak is not meaningful for $a-\mathrm{C}:(\mathrm{H})$ films. The existence of the $D$ peak is considered to be evidence for benzene or condensed benzene rings in amorphous (hydrogenated) carbon films. We also show that the $G$ peak is not necessarily composed only of the $E_{2 g_{2}}$ mode of graphite but also of $s p^{2} \mathrm{C}=\mathrm{C}$ stretch vibrations.

A possible explanation for the position of the $G$ peak and its width will be discussed. The article also tries to underline the importance of the $I_{D} / I_{G}$ intensity ratio and the $G$ linewidth. Only by taking both the $I_{D} / I_{G}$ intensity ratio and the $G$ linewidth can an estimate of the benzene cluster sizes in carbon films be obtained.

\section{EXPERIMENTAL DETAILS}

The films studied in this article were prepared by a magnetron and a magnetically field enhanced plasma deposition system (MEPD). The films prepared in the unbalanced magnetron source were amorphous carbon films (with hydrogen incorporation lower than 2 at. $\%$ as determined by ${ }^{15} \mathrm{~N}$ nuclear resonance method) and were deposited by sputtering of a graphite target in an $\mathrm{Ar}$ atmosphere at a pressure of $1.5 \times 10^{-3}$ mbar at temperatures below $50^{\circ} \mathrm{C}$. For two different argon ion/neutral carbon ratios of five and ten, carbon films were deposited at different argon ion energies on crystalline silicon substrates. The films reveal densities up to 3.1 $\mathrm{g} / \mathrm{cm}^{3}$ and $s p^{3}$ ratios of up to $87 \%$ as determined by EELS measurements. ${ }^{21}$ Carbon films with such a high $s p^{3}$ ratio are often referred to as highly tetrahedral amorphous carbon films ( $t a-C)$. The deposition mechanism and other physical properties are explained in more detail elsewhere. $^{21}$

The films prepared by a MEPD were performed by an inductively coupled $13.56 \mathrm{MHz}$ acetylene discharge at different pressures ranging from $7 \times 10^{-4}$ up to $2 \times 10^{-3}$ mbar. The $a$-C:H films were deposited onto a water-cooled grounded substrate holder. These films contain about 29-32 at. \% hydrogen depending on the pressure of the discharge. The applied magnetic field was $500 \mathrm{G}$ at the substrate surface as determined by Hall probe measurements. More details about the films and the deposition system are found elsewhere. $^{22}$

The Raman measurements were carried out on a Raman apparatus using the $515 \mathrm{~nm}$ line of an Ar ion laser focused to
TABLE I. Before fitting of the carbon films prepared by magnetron sputtering the background of the Raman spectrum has to be subtracted. Therefore four Gaussians centered at the given wavelengths had to be subtracted. The original Raman spectrum of one film can be seen in Fig. 1(a) together with the background spectrum. Figure 1(b) shows the resulting Raman spectrum after the subtraction of the background together with the final fit in the discussed region between 1000 and $1700 \mathrm{~cm}^{-1}$.

\begin{tabular}{cl}
\hline $\begin{array}{c}\text { Background peaks } \\
\left(\mathrm{cm}^{-1}\right)\end{array}$ & \multicolumn{1}{c}{ Assigned to: } \\
\hline 800 & Hydrogen free carbon structure (Ref. 6) \\
1950 & $\begin{array}{l}\text { Second-order combination scattering of two } \\
\text { main structures at } 550 \text { and } 1550 \mathrm{~cm}^{-1} \\
\text { (Ref. 23) }\end{array}$ \\
& $\begin{array}{l}\text { Two phonon excitation from the } 1100 \text { to } \\
1300 \mathrm{~cm}^{-1} \text { region (Ref. 8) }\end{array}$ \\
& Combination of the zone center $1580 \mathrm{~cm}^{-1}$ \\
2420 & $\begin{array}{l}\text { mode and the zone boundary } 1355 \mathrm{~cm}^{-1} \\
\text { mode (Ref. } 24) \\
\text { or combination of the density of states } \\
\text { feature at 1620 cm } \mathrm{cm}^{-1} \text { and the zone boundary } \\
1355 \mathrm{~cm}^{-1} \text { mode (Ref. 8) }\end{array}$ \\
\hline \hline
\end{tabular}

$2 \mathrm{~mm}^{2}$. The laser power was $500 \mathrm{~mW}$ and the spectral resolution of the apparatus was $8 \mathrm{~cm}^{-1}$. Typical data acquisition times were in the range of $25 \mathrm{~min}$. The spectra were recorded in the range of $80-3000 \mathrm{~cm}^{-1}$ in order to allow reliable fitting (Table I). In the case of the MEPD deposited $a-\mathrm{C}: \mathrm{H}$ films the Raman spectra have been fitted by two Gaussians allowing all the fit parameters to vary. In the case of films deposited by magnetron sputtering combined with ion plating (MS/IP) most of the films could not be fitted by two Gaussians (except those deposited at low Ar plating energy). To obtain a good fit it was necessary to use four Gaussians (Table II). Here all the fit parameters such as linewidths, positions, and areas were allowed to vary [Fig. 1(b)]. Other Raman data has been taken from the literature. Figure 1(b) shows Raman features which are due to a highly disordered carbon structure.

\section{RESULTS AND DISCUSSION}

Tuinstra and Koenig ${ }^{7}$ found that the Raman intensity of the $I_{D} / I_{G}$ is inversely proportional to the graphite crystallize size $L_{a}$ :

$$
\frac{1}{L_{a}} \propto \frac{I_{1355}}{I_{1575}}=\frac{I_{D}}{I_{G}},
$$

where $L_{a}$ is the graphite crystallite size as determined by $\mathrm{x}$ ray. Equation (1), although valid for graphite, has so far not been proven to be valid for amorphous carbon and hydrogenated amorphous carbon films. Tamor et al. ${ }^{30}$ showed by comparison with optical measurements that Eq. (1) cannot be applied to amorphous carbon. Therefore the analogy of amorphous carbon to graphite is not straightforward. Knight and White ${ }^{28}$ presented data which when extrapolated showed an $I_{D} / I_{G}$ ratio of four for a graphitic cluster size of $10 \AA$. Thus, the experiment places an upper limit of $10-20 \AA$ for 
TABLE II. Raman features in the range between 1000 and $1700 \mathrm{~cm}^{-1}$ are listed. Possible interpretations of these features are given. The Raman features denoted by $(*)$ are used for fitting.

\begin{tabular}{|c|c|}
\hline $\begin{array}{c}\text { Raman feature } \\
\mathrm{cm}^{-1}\end{array}$ & Possible interpretation \\
\hline $1180(*)$ & $\begin{array}{l}\text { Nanocrystalline diamond (Ref. } 25) \\
\text { hexagonal diamond (Refs. } 12,26 \text {, and } 27 \text { ) or } \\
\text { sp }{ }^{3} \text {-rich phase (Refs. } 12 \text { and } 13 \text { ) }\end{array}$ \\
\hline 1305 & Hexagonal diamond (Refs. 27 and 28) \\
\hline 1332 & Cubic diamond \\
\hline $1350(*)$ & $\begin{array}{l}D \text { peak of microcrystalline graphite (Ref. 6) or } \\
\text { alternating ring stretch vibration in benzene } \\
\text { or condensed benzene rings }\end{array}$ \\
\hline $1490(*)$ & $\begin{array}{l}\text { Semicircle ring stretch vibration of benzene } \\
\text { or condensed benzene rings or } \\
\text { in the case of } a-\mathrm{C}: \mathrm{H} \text { contributions of } \mathrm{C}-\mathrm{H} \\
\text { vibrations at } 1500 \mathrm{~cm}^{-1} \text { (Ref. 29) } \\
\text { or contributions from the phonon density of } \\
\text { states in finite-size crystals of graphite (Ref. 8) }\end{array}$ \\
\hline $1580(*)$ & $\begin{array}{l}G \text { peak of graphite (Ref. 6) or } \\
s p^{2} \text { stretch vibration in benzene or condensed } \\
\text { benzene rings } \\
\text { or } s p^{2} \text { stretch vibration of olefinic/conjugated } \\
\text { chains }\end{array}$ \\
\hline
\end{tabular}

the graphitic cluster size in amorphous carbon films. Such small cluster sizes evoke the question of the origin of the $D$ and $G$ peak in amorphous carbons.

\section{A. $G$ peak and $D$ peak in amorphous (hydrogenated) carbon films}

Consider the $G$ peak. Single benzene rings exhibit a Raman peak at $1588 \mathrm{~cm}^{-1}$ [Fig. 2(a)]. This feature is valid for benzene, all aromatic rings, and condensed benzene rings. A possible contribution to the $G$ peak therefore may arise from the $\mathrm{C}=\mathrm{C} s p^{2}$ stretch vibrations of olefinic or conjugated carbon "chains", (or first-order scattering from features in the density of states of a graphite network) at about $1620 \mathrm{~cm}^{-1}$, too. Thus, the existence of a $G$ peak in the Raman spectrum does not necessarily prove that the $a$-C film consists only of graphite or fused benzene rings. The $G$ peak might as well have its origin in olefinic chains in the carbon film. Benzene rings show a further Raman feature at $1486 \mathrm{~cm}^{-1}$ [Fig. 2(b) ]. ${ }^{29}$ A peak centered at $1490 \mathrm{~cm}^{-1}$ can be attributed to semicircle stretching of carbon atoms in single aromatic rings or fused aromatic rings. ${ }^{29,31}$ Another possible explanation for the peak at $1470 \mathrm{~cm}^{-1}$ (in finite-size crystals of graphite) was given by Nemanich and Solin, ${ }^{8}$ who attributed this peak to contributions from the phonon DOS.

In the case of the $D$ peak, interaction force constant effects have to be considered. ${ }^{29}$ Normally, the simple valence force field is used to calculate vibration frequencies. If two or more oscillators in a molecule or solid are distorted from equilibrium, they may be able to affect each other's restoring forces to some extent, due to changes in the electronic structure. A more general valence force field considers the total restoring force on one oscillator to be equal to a force constant times its own distortion plus a second force constant

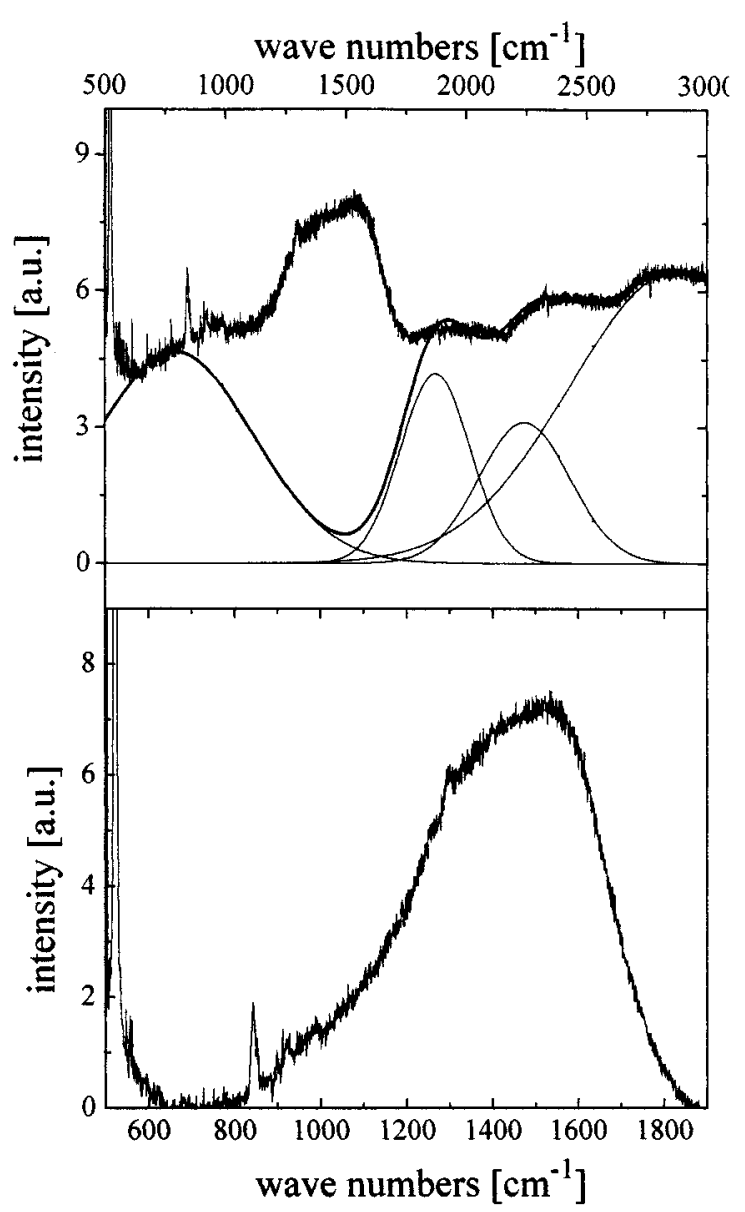

FIG. 1. (a) Original Raman spectrum of MS/IP deposited $a-\mathrm{C}$ and its background from Table I, (b) Raman spectrum of (a) subtracted by the background fit to the data for MS/IP deposited $a$-C. Gaussian line shapes have been used as Lorentzian line shapes would only be expected in the presence of crystalline inclusions in the amorphous carbon films. The Raman features are expected to consist of multiple lines as shown in Fig. 2(d) which is also an indication for Gaussian line shapes. a)

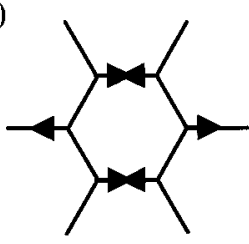

c)<smiles>c1ccccc1</smiles><smiles>c1ccccc1</smiles>

b)

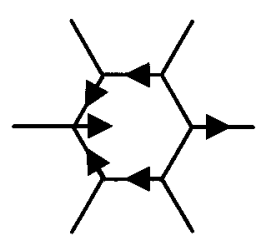

d)

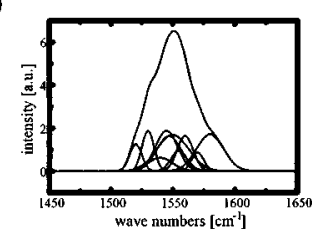

FIG. 2. (a) Carbon stretch vibrations in benzene centered at $1588 \mathrm{~cm}^{-1}$, (b) semicircle stretch vibrations in benzene at $1486 \mathrm{~cm}^{-1}$, (c) alternating ring stretch in benzene centered at $1311 \mathrm{~cm}^{-1}$ due to interaction force constant effects (Ref. 29), (d) superposition of different Raman active modes leading to broader effective linewidths 


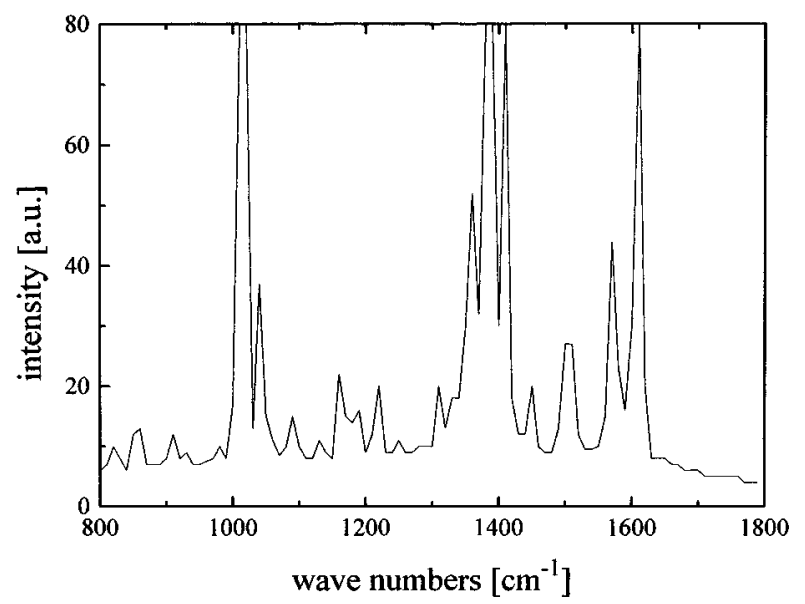

FIG. 3. Raman spectrum 1,2,3,4-tetraphenylnaphthalene after Ref. 32. Strong Raman scattering is observed at $1380 \mathrm{~cm}^{-1}$. This feature is stronger than the $1600 \mathrm{~cm}^{-1}$ stretch vibrations of the $s p^{2}$ modes. This molecule exhibits a significant " $I_{D} / I_{G}$ ratio."

times the distortion of a second oscillator. The second force constant is called the interaction force constant and can be either positive or negative. The alternating ring stretch of benzene is illustrated in Fig. 2(c). During this vibration, the electronic structure changes and approaches the Kekule benzene structure where electrons partially slip away from stretched bonds into contracted bonds. This leads to the frequency of the alternating ring stretch vibration of benzene to $1311 \mathrm{~cm}^{-1}$, much lower than that predicted from the simple valence force field model $\left(1700 \mathrm{~cm}^{-1}\right)$. For a more detailed discussion see Colthup et al. ${ }^{29}$ The existence of a peak centered at about $1350 \mathrm{~cm}^{-1}$ proves the existence of aromatic or benzene clusters incorporated in the amorphous (hydrogenated) carbon films. Nevertheless, if a Raman spectrum of a carbon film reveals a peak at $1350 \mathrm{~cm}^{-1}$ this peak may as well have its origin in the phonon DOS contribution of highly disordered graphitic structures ${ }^{8}$ (see also Table II).

The Raman spectrum of the molecule 1,2,3,4-tetraphenylnaphthalene ${ }^{32}$ is shown in Fig. 3. Similar Raman spectra of molecules such as 1,4-diphenylbutadiene, 2,6dimethylnaphthalene, acenaphthene, fluorene, naphthalene, ${ }^{32,33}$ etc., all exhibit typical Raman features at about 1600,1490 , and $1350 \mathrm{~cm}^{-1}$. In addition to these molecules containing a large amount of hydrogen, compared to $a-\mathrm{C}: \mathrm{H}$, the spectra prove that even small aromatic cluster sizes can account for the Raman features normally observed in amorphous carbon and hydrogenated amorphous carbon.

\section{B. Line broadening effects in amorphous carbon}

In principle there may be four significant reasons for line broadening of the $G$ line feature in $a-\mathrm{C}: \mathrm{H}$ films; namely the cluster size, the cluster distribution, the influence of the stress in the films, and broadening due to chemical bonding.

Nemanich et al. ${ }^{12}$ and Shroder, Nemanich, and Glass ${ }^{13}$ found that the relative concentration and crystallite size are crucially important in the analysis of Raman spectra. They also showed that the vibrational modes of small crystallites

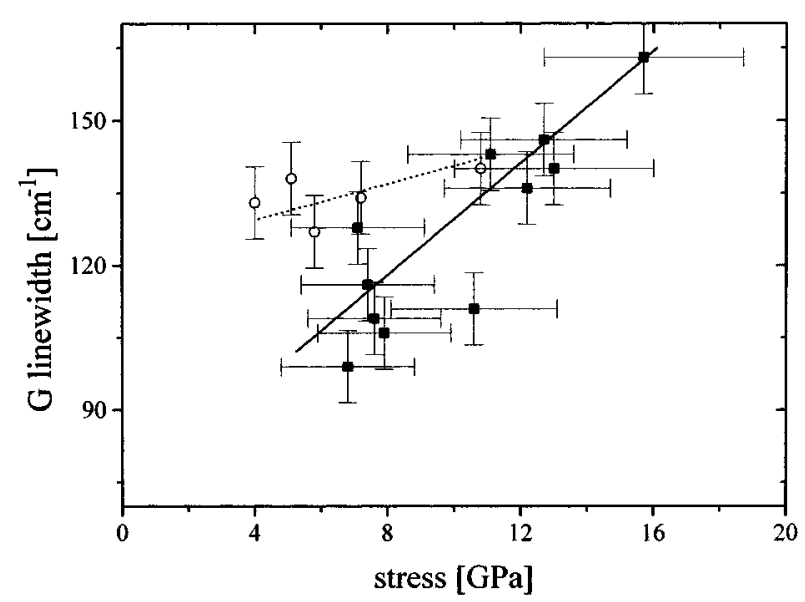

FIG. 4. $G$ linewidth vs stress for $a-\mathrm{C}$ films deposited by MS/IP technique (ם) and $a-\mathrm{C}: \mathrm{H}$ films deposited by MEPD technique (O).

can be described as phonons with an uncertainty in the wave vector $(\Delta k \propto 2 \pi / d$, where $d$ is the domain size).

Therefore, it is possible that the cluster size may also influence the linewidth of the $G$ feature. Nemanich et al. ${ }^{12}$ and Schroder, Nemanich, and Glass ${ }^{13}$ also discussed the absorption depths and phonon decay lengths for the constituent material as being larger than the domain size and as a result the Raman spectra will be a superposition if the phonons are confined to different regions. This is considered to be the case in $a-\mathrm{C}$ and $a-\mathrm{C}: \mathrm{H}$ due to the existence of various numbers of clusters of different sizes and different environments. Thus a superposition of the different Raman modes will occur [shown schematically in Fig. 2(d)] and broader linewidths are expected. The influence of the stress on the $G$ linewidth of the $a-\mathrm{C}$ and $a-\mathrm{C}: \mathrm{H}$ films is shown in Fig. 4. This figure reveals that stress cannot be ruled out as a line broadening factor since this also is a measure of strain in the surroundings of the benzene clusters and $s p^{2}$ bonding. For graphite, Sakata et al. ${ }^{34}$ found that the half-width of the 1580 $\mathrm{cm}^{-1}$ peak increases linearly with increasing stress. Similar to the findings of Sakata et $_{\text {al. }}{ }^{34}$ we too find a linear dependence of the $G$ linewidth with stress, with the linewidth found for the $a-\mathrm{C}$ and $a-\mathrm{C}: \mathrm{H}$ films being much broader than those of graphite. From Fig. 4 it may be deduced that the latter ( $a-\mathrm{C}: \mathrm{H}$ films) traces a different slope in comparison to the data for amorphous carbon films. If comparisons were made to the variation of stress in graphite, ${ }^{34}$ it would indicate that the hydrogen causes changes in the elastic constants in amorphous carbon films.

Thus, all four effects (size, distribution, stress, chemical environment) may contribute to the broadening of the $G$ linewidth. In principle these reasons may also be true for the linewidth of the $D$ line.

\section{C. $I_{D} / I_{G}$ ratio in amorphous carbon}

Examining the Raman spectrum of the simple symmetric 1,2,3,4-tetraphenylnaphthalene (Fig. 3) it can be seen that there is a more intense $D$ peak feature compared to the $G$ peak feature although the benzene clusters are small. There exist a lot of border regions of the benzene clusters which are responsible for the $D$ peak feature. It is now possible to discuss the observed maximum of the $I_{D} / I_{G}$ ratio as a func- 


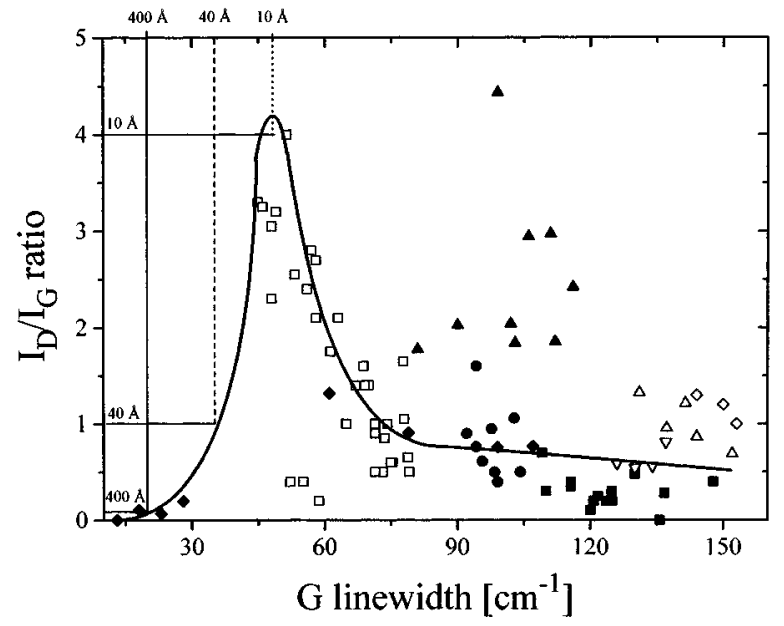

FIG. 5. $I_{D} / I_{G}$ ratio vs $G$ linewidth for $a$-C:H $\square$ (Ref. 6), MEPD deposited $a-\mathrm{C}: \mathrm{H} \Delta, a-\mathrm{C}: \mathrm{H}$ deposited by magnetic confinement $\nabla$ (Ref. 35), $a-\mathrm{C}: \mathrm{H}$ deposited by Mariotto et al. $\diamond$ (Ref. 36), sputtered $a$-C $\bullet$ (Ref. 6), $i$-C or $t a$-C — (Ref. 6), MS/IP deposited $t a-\mathrm{C} \Delta$, various graphite and other carbons by Knight et al. (Ref. 28). We propose that the $D$ peak for carbon films with a $G$ linewidth smaller than $45 \mathrm{~cm}^{-1}$ is dominated by microcrystalline graphite inclusions whereas the $D$ peak for $a-\mathrm{C}(: \mathrm{H})$ films with $G$ linewidths broader than $45 \mathrm{~cm}^{-1}$ is mostly dominated by small aromatic clusters with cluster sizes lower than $10 \AA$.

tion of the cluster sizes in the $a-\mathrm{C}$ and $a-\mathrm{C}: \mathrm{H}$ films shown in Fig. 5. Tuinstra and Koenig ${ }^{7}$ did not find a $D$ peak in ideal graphite. If this ideal graphite is now assumed to be more and more disturbed by $s p^{3}$ bonded carbon, the $D$ peak will develop and rise in magnitude in the Raman spectrum. When the material is composed entirely of $s p^{3}$ bonded carbon (and/or olefinic chains connected by $s p^{3}$ bonded carbon) the $D$ peak should not be detected in this model because there exist no benzene clusters that gave rise to the disordered regions which are responsible for the $D$ peak, i.e., in a completely $s p^{3}$ network the $I_{D} / I_{G}$ ratio will tend to zero. This is observed by Prawer et al. ${ }^{37}$ in $t a-\mathrm{C}$ films prepared by mass selected ion beams having $s p^{3}$ ratios of about $80 \%$. According to the model proposed the $I_{D} / I_{G}$ ratio will go through a maximum which is dependent on the cluster sizes and distributions. Using the extrapolation of Knight and White ${ }^{28}$ and using the experimental results that a maximum to the ratio of four was found, the optimum cluster size for a maximum $I_{D} / I_{G}$ ratio is estimated to be $10 \AA$. But still the $I_{D} / I_{G}$ ratio is generally a measure for the zone edges or border phonons ${ }^{38}$ of the clusters (which depend on cluster sizes and distributions).

Tamor et al. ${ }^{30}$ concluded from their measurements that the $I_{D} / I_{G}$ ratio is not a useful indicator of graphitic clusters in amorphous carbon films. The Raman results so far confirm the cluster model of Wada, Gaczi, and Solin ${ }^{14}$ and Robertson and O'Reilly. ${ }^{16}$ But Raman spectroscopy does not necessarily prove the existence of large cluster sizes that are discussed in these models. Ehrhardt et al. ${ }^{19}$ and Kleber et al. ${ }^{20}$ found cluster sizes of up to 5 fused benzene rings in their $a-\mathrm{C}: \mathrm{H}$ films as derived from ESR.

Since the $G$ linewidth is partly determined by the domain or size of the (fused) benzene rings, the $I_{D} / I_{G}$ ratio is plotted versus $G$ linewidth in Fig. 5 for various $a$-C and
$a-\mathrm{C}: \mathrm{H}$ films also using the data set of Tamor and Vassel. ${ }^{6}$ Further data of Knight and White, ${ }^{28}$ Silva et al. ${ }^{35}$ and Mariotto et $_{\text {al. }}{ }^{36}$ are used. All named authors used the $5145 \AA$ excitation line, so that their results can be compared. Here the difference between graphite, microcrystalline graphite, chemical vapor deposited $a-\mathrm{C}: \mathrm{H}$, sputtered $a-\mathrm{C}, t a-\mathrm{C}$ (tetrahedrally bonded amorphous carbon) deposited by laser ablation, and MEPD deposited $a-\mathrm{C}: \mathrm{H}$ films can be clearly seen. The line through these points is for leading the eye. The carbon films deposited by MS/IP will be discussed later. In the case of microcrystalline graphite the small linewidth and low $I_{D} / I_{G}$ ratio are good indicators for the crystallinity of the samples. As the $G$ linewidth gets broader there is a maximum in the $I_{D} / I_{G}$ ratio of four. In order to get a better survey the graphitic cluster sizes, those from Tuinstra and Koenig ${ }^{7}$ and Knight and White ${ }^{28}$ are also included on the $I_{D} / I_{G}$ axis and the bottom line of the $G$-linewidth axis.

When the $G$ linewidth for amorphous carbon films and amorphous hydrogenated carbon films increases above a value of $50 \mathrm{~cm}^{-1}$ it is observed (Fig. 5) that the $I_{D} / I_{G}$ ratio decreases. As discussed previously [see Eq. (1)], if the $G$ linewidth increases beyond $50 \mathrm{~cm}^{-1}$, it results in a reduction in the $I_{D} / I_{G}$ ratio. From the work of Tuinstra and Koenig ${ }^{7}$ we see that the maximum in the $I_{D} / I_{G}$ peak value of 4 corresponds to an effective graphitic cluster size for carbon films of $10 \AA$. Therefore, when the $G$ linewidth increases beyond $50 \mathrm{~cm}^{-1}$, the resulting decrease in the $I_{D} / I_{G}$ ratio would be indicative of a cluster size smaller than $10 \AA$ according to Eq. (1). This result is confirmed by the extrapolated values for microcrystalline graphite by Knight and White. ${ }^{28}$ The data points in Fig. 5 lead to the conclusion that the fused benzene clusters in $a-\mathrm{C}: \mathrm{H}$ deposited by chemical vapor deposition are larger than in sputtered $a$-C. Recent transmission electron microscope results by Davis et al. ${ }^{40}$ on $a$-C:H superlattice structures show there to be no evidence of clusters greater than $5 \AA$ in rf plasma deposited $a$-C:H films. The Raman data for amorphous sputtered carbon reveal that only a minor fraction of ordered sixfold rings exists. ${ }^{41}$ If there were larger clusters inside the sputtered carbon films the $G$ linewidth should be smaller and the $I_{D} / I_{G}$ ratio should be higher. In the case of ion-carbon $(i-\mathrm{C})$ or highly tetrahedral amorphous carbon $(t a-\mathrm{C})$ the $I_{D} / I_{G}$ ratio together with the $G$ linewidth indicates the presence of little benzene (cluster) inclusions within the films. The $a$-C:H films deposited by the MEPD also possess low $I_{D} / I_{G}$ ratio and broad $G$ linewidth. ${ }^{22,37}$ This is also an indication for small clusters in the films. The optical gap of these films is quite high $(2 \mathrm{eV})$ which also reinforces the interpretation of cluster sizes as derived from the $I_{D} / I_{G}$ ratio and $G$ linewidth.

The $t a-\mathrm{C}$ films deposited by the unbalanced magnetron deviate from the data of the other carbon films. In the authors opinion the high stress in the films (up to $20 \mathrm{GPa}$ measured by the bending beam method using Stoney's equation) is responsible for the broad $G$ linewidth. In these films the $I_{D} / I_{G}$ ratio alone indicates an effective cluster size of about $10 \AA$ A. Thus, only the knowledge about both the $I_{D} / I_{G}$ ratio and the $G$ linewidth allows a rough estimate of the cluster size in amorphous (hydrogenated) carbon films. Having in- 


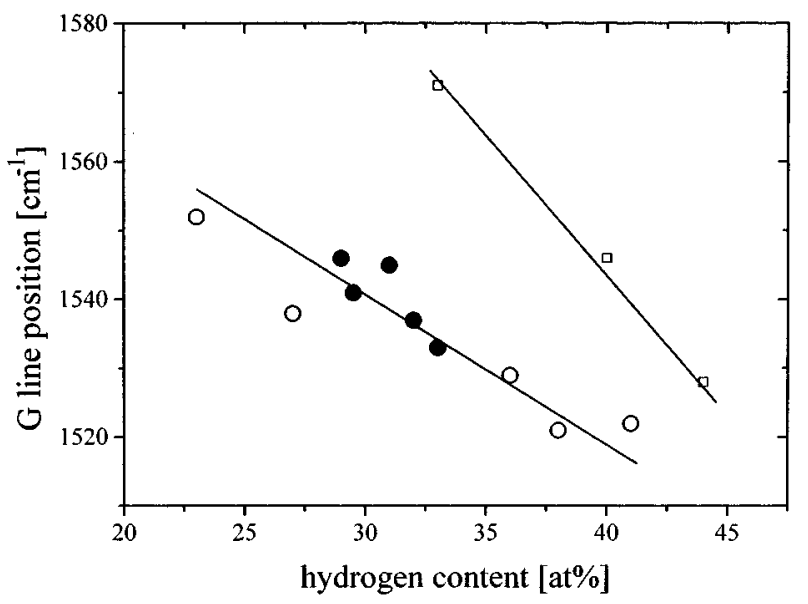

FIG. 6. $G$ position vs hydrogen content for $a-\mathrm{C}: \mathrm{H}$ (Ref. 6) deposited using benzene $\mathrm{O}, a-\mathrm{C}: \mathrm{H}$ deposited by MEPD technique $\mathrm{O}, \mathrm{a}-\mathrm{C}: \mathrm{H}$ deposited by methane $\square$ (Ref. 6).

formation only about the $I_{D} / I_{G}$ ratio does not give reliable information with regard to the cluster sizes in carbon films.

\section{Positions of the $G$ and $D$ peak of amorphous (hydrogenated) carbon films}

The position of the $G$ peak is more complicated. The dependence of the $G$ position on $H$ content for some $a-\mathrm{C}: \mathrm{H}$ films is shown in Fig. 6. This figure reveals that the $G$ position depends on the local environment of the benzene clusters and $s p^{2}$ bonding. Ager et al. ${ }^{42}$ further found a strong dependence of the $G$ position on stress in $t a-C$ films deposited by filtered cathodic vacuum arc. They showed that by delaminating the highly stressed $t a-\mathrm{C}$ films from crystalline silicon (allowing the films to relax) the $G$ position may drop by about $20 \mathrm{~cm}^{-1}$.

Another possible feature which might influence the $G$ line position is the peak at $1486 \mathrm{~cm}^{-1}$ which has already been attributed to semicircle stretch vibrations in benzene or benzene clusters. Wagner et al. ${ }^{38}$ and Ramsteiner et al. ${ }^{43}$ found an interference-enhanced Raman scattering in hard amorphous carbon films, while the position of the $G$ peak and the intensity ratio $I_{D} / I_{G}$ of the $D$ and $G$ peaks depended on the incident photon energy. They explained this by a twophase model in which the Raman spectrum is a superposition of two distinct vibronic Raman spectra. They attributed phase 1 (revealing a Raman peak between 1300 and 1500 $\mathrm{cm}^{-1}$ ) to a $s p^{2}$ network including $s p^{3}$ bonds and phase 2 (revealing a Raman peak at about $1600 \mathrm{~cm}^{-1}$ ) to $s p^{2}$ clusters. They assumed that as the photon energy changes both phases have distinct contributions to the overall Raman scattering thus changing the $G$ position and $I_{D} / I_{G}$ intensity ratio. This model has to be extended in a similar manner such that $a-\mathrm{C}: \mathrm{H}$ or $a-\mathrm{C}$ is not a two-phase system but an $n$-phase system, because the absorption depths and phonon decay lengths for the constituent material are expected to be larger than the domain size (optical skin depth in graphite amounts to $600 \AA$ for light scattering at $488 \mathrm{~nm})^{34}$ of the condensed benzene rings. ${ }^{12,13}$ The feature at about $1580 \mathrm{~cm}^{-1}$, as well as the feature at $1486 \mathrm{~cm}^{-1}$ (their possible origins are listed

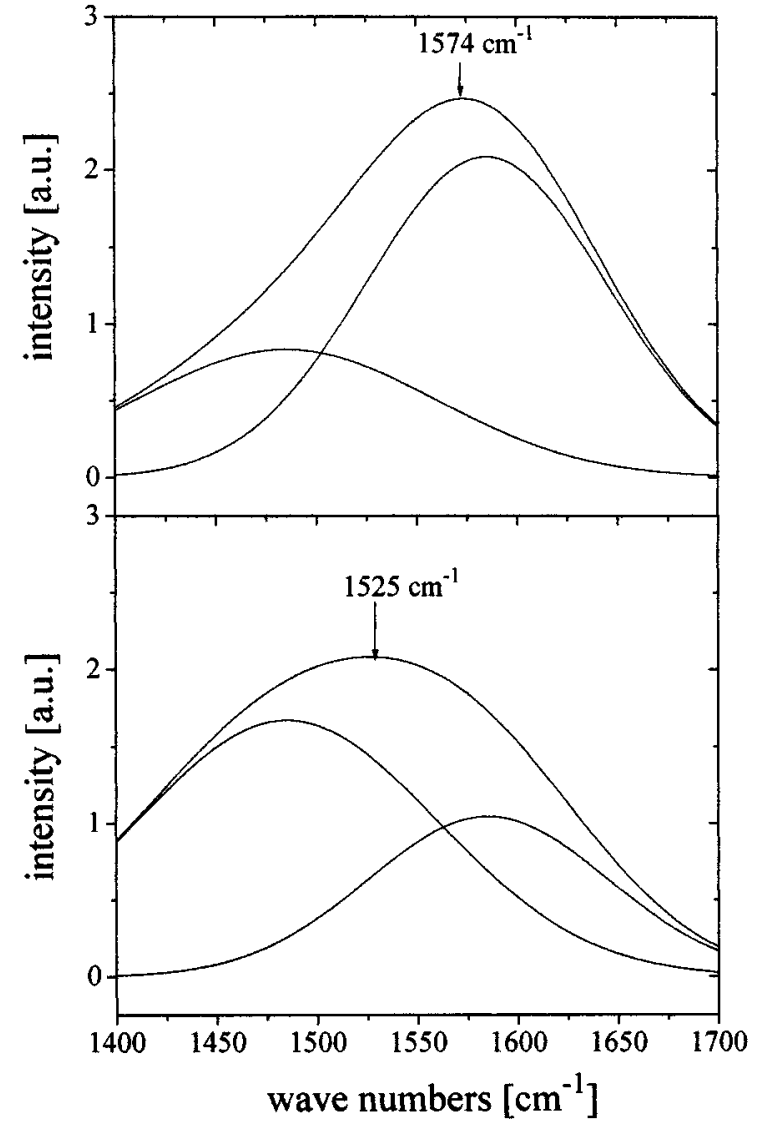

FIG. 7. Superposition model of $1486 \mathrm{~cm}^{-1}$ Raman feature and $1580 \mathrm{~cm}^{-1}$ Raman feature in order to explain the shift of the $G$-peak position. The different intensities of these two Raman features determine the observed $G$ position.

in Table II), may superimpose and thus affect the $G$ position depending on the intensity of each feature (Fig. 7). Thus, the $G$ position is determined by several factors such as the hydrogen content and stress. The variation of the $D$ position versus $G$ position is shown in Fig. 8. This does not mean that

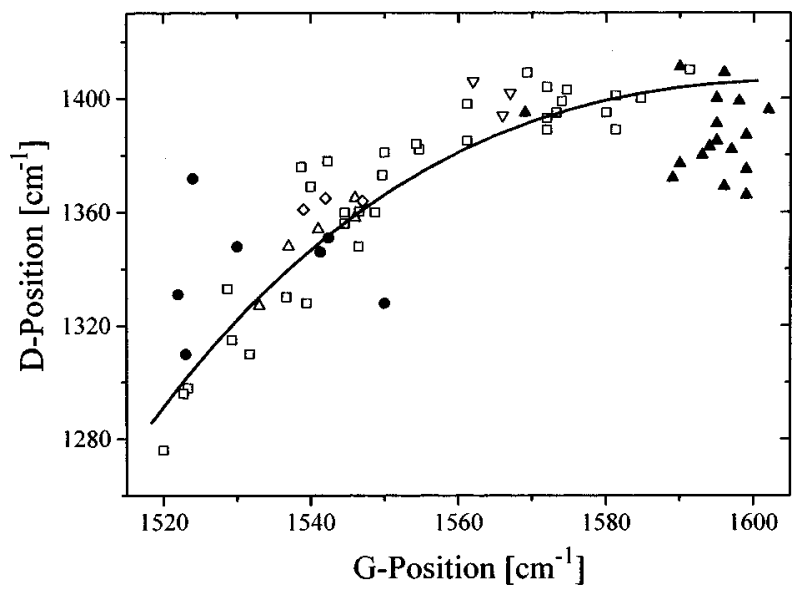

FIG. 8. $D$ position vs $G$ position for $a$-C:H $\square$ (Ref. 6), MEPD deposited $a-\mathrm{C}: \mathrm{H} \Delta, a-\mathrm{C}: \mathrm{H}$ deposited by magnetic confinement $\nabla$ (Ref. 39), $a-\mathrm{C}: \mathrm{H}$ deposited by Mariotto et al. $\diamond$ (Ref. 36), sputtered $a$-C $\bullet$ (Ref. 6), MS/IP deposited $t a-\mathrm{C} \Delta$. 


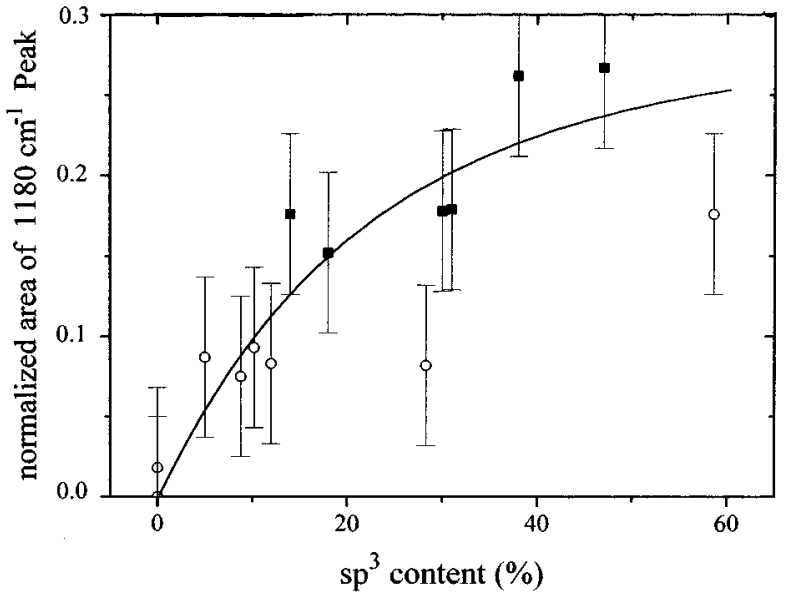

FIG. 9. Normalized area of the $1180 \mathrm{~cm}^{-1}$ Raman peak vs $s p^{3}$ content in the carbon films deposited by MS/IP technique.

the $D$ position is a function of the $G$ position. Figure 8 shows that in principle the same arguments that hold for the $G$ position may also hold for the $D$ position. It illustrates that for example stress in the films shifts the $G$ position as well as the $D$ position. Figure 8 also reveals that there is a strong correlation between the $G$ position and $D$ position on the type of the films which was already shown by Tamor et al. ${ }^{6}$

\section{E. Raman feature at $1180 \mathrm{~cm}^{-1}$ for carbon films deposited by the magnetron}

In the films deposited by the MS/IP technique we were not able to obtain good fits just by considering the $G$ and $D$ peaks. Acceptable fits could only be reached by adding two extra peaks at approximately 1180 and $1490 \mathrm{~cm}^{-1}$. The peak at $1490 \mathrm{~cm}^{-1}$ has already been discussed. Together with the $1180 \mathrm{~cm}^{-1}$ peak a rather small and narrow peak at 1305 $\mathrm{cm}^{-1}$ is observed for films with high $s p^{3}$ content. The peak at $1180 \mathrm{~cm}^{-1}$ is very broad with a bandwidth of $200 \mathrm{~cm}^{-1}$. One of the components that causes such a broadening is the high compressive stress present in the films.

A peak at $1140 \mathrm{~cm}^{-1}$ was attributed to nanocrystalline diamond by Yarbrough et al. ${ }^{25}$ Silva et al. ${ }^{26}$ assigned a peak at $1170 \mathrm{~cm}^{-1}$ to hexagonal diamond based on glancing angle $\mathrm{x}$-ray and Raman results. Nemanich et al. ${ }^{12}$ stated that this peak could be either due to hexagonal diamond or a $s p^{3}$-rich phase or nanocrystalline diamond. Shroder, Nemanich, and Glass $^{13}$ attributed this peak to $s p^{3}$ carbon vibrations. Kohanoff ${ }^{27}$ predicted by theoretical considerations two peaks at 1237 and $1306 \mathrm{~cm}^{-1}$ to be due to hexagonal diamond. The peaks we observe at 1180 and $1305 \mathrm{~cm}^{-1}$ cannot be confirmed as that belonging to hexagonal diamond, because Raman scattering alone is not adequate to prove that hexagonal diamond inclusions are present in these films. Nevertheless, the normalized area of the $1180 \mathrm{~cm}^{-1}$ peak (normalized to the Raman signal between 1000 and $1650 \mathrm{~cm}^{-1}$ ) is plotted against $s p^{3}$ content of the films in Fig. 9. Since the error bars are very large Fig. 9 just shows a trend. The results emphasize that the peak at $1180 \mathrm{~cm}^{-1}$ is more pronounced in the presence of $s p^{3}$ stretching in the carbon films. This is so far only valid for $t a-\mathrm{C}$ films prepared by the MS/IP technique. It may not hold for other forms of carbon films. It should be noted that in the case of hexagonal diamond a "puckered" $s p^{3}$ bond structure is present and the puckering could be caused by excessive stress on a cubic $s p^{3}$ bonded crystalline structure as recently shown by Kohanoff. ${ }^{27}$

\section{SUMMARY AND CONCLUSION}

The complexity of the Raman spectra of amorphous carbon is discussed in this paper. Raman interpretation has to be performed with great care due to information being lost as a result of the very broad $D$ line and $G$ line features. This makes the determination of cluster sizes and distributions based only on Raman spectroscopy very difficult. Even using both the $I_{D} / I_{G}$ ratio and the $G$ linewidth only a crude estimate of cluster sizes can be elucidated.

This paper also shows that the $G$ line feature and $D$ line feature do not require large cluster sizes as initially predicted. ${ }^{14,16}$ The $G$ line may even be formed by olefinic $s p^{2}$ bonds alone as well as $s p^{2}$ stretch vibrations in benzene rings. The $D$ line can be explained by interaction force constant effects and does not require large clusters either. The $G$ linewidth is determined by cluster size, the cluster size distribution, and stress in the carbon films. It is impossible to predict the dominating line broadening factor, namely stress or cluster size. The $G$ line position is determined by several factors, namely, the hydrogen content, local environment of the benzene clusters, the cluster size and their distribution, stress, and the superposition of the semicircle stretching at $1486 \mathrm{~cm}^{-1}$ with the Raman modes above $1500 \mathrm{~cm}^{-1}$.

Further it has been shown that the $1180 \mathrm{~cm}^{-1}$ line correlates to the $s p^{3}$ content in the films prepared by magnetron sputtering combined with $\mathrm{Ar}$ ion plating. So far this is the only peak in the Raman spectrum (beside the diamond peak at $1332 \mathrm{~cm}^{-1}$ ) which can be attributed to $s p^{3}$ bonded carbon. All other features observed by Raman in the 1000-1650 $\mathrm{cm}^{-1}$ region are believed to have their origin in $s p^{2}$ bonded carbon.

\section{ACKNOWLEDGMENTS}

This work is sponsored by the Deutsche Forschungsgemeinschaft Project No. Eh 23/29-1. S.R.P.S. wishes to thank the Royal Society for financial assistance (GRA 574006).

${ }^{1}$ S. Aisenberg and R. Chabot, J. Appl. Phys. 42, 2953 (1971).

${ }^{2}$ D. McKenzie, R. McPhedran, N. Savvides, and L. Botten, Philos. Mag. B 48, 341 (1983).

${ }^{3}$ J. Robertson, Adv. Phys. 35, 317 (1986).

${ }^{4}$ H. Tsai and D. Bogy, J. Vac. Sci. Technol. A 5, 3287 (1987).

${ }^{5}$ J. C. Angus, Thin Solid Films 216, 126 (1992).

${ }^{6}$ M. Tamor and W. Vassel, J. Appl. Phys. 76, 3823 (1994).

${ }^{7}$ F. Tuinstra and J. Koenig, J. Chem. Phys. 53, 1126 (1970).

${ }^{8}$ R. Nemanich and S. Solin, Phys. Rev. B 20, 392 (1979).

${ }^{9}$ R. J Nemanich, G. Lucovsky, and S. A. Solin, Solid State Commun. 23, 117 (1977).

${ }^{10}$ P. Lespade, R. Al-Jishi, and M. Dresselhaus, Carbon 20, 427 (1982).

${ }^{11}$ N. Wada and S. A. Solin, Physica B 105, 353 (1981).

${ }^{12}$ R. Nemanich, J. Glass, G. Lucovsky, and R. Shroder, J. Vac. Sci. Technol. A 6, 1783 (1988).

${ }^{13}$ R. Shroder, R. Nemanich, and J. Glass, Phys. Rev. B 41, 3738 (1990).

${ }^{14}$ N. Wada, P. Gaczi, and S. Solin, J. Non-Cryst. Solids 35-36, 543 (1980). 
${ }^{15}$ D. Beeman, J. Silverman, R. Lynds, and M. Anderson, Phys. Rev. B 30, 870 (1984).

${ }^{16}$ J. Robertson and E. O'Reilly, Phys. Rev. B 35, 2946 (1986).

${ }^{17}$ R. Dillon, J. Wodam, and V. Katkanant, Phys. Rev. B 29, 3482 (1984).

${ }^{18}$ F. Li and J. Lannin, Appl. Phys. Lett. 61, 2116 (1992).

${ }^{19}$ H. Ehrhardt, R. Kleber, A. Krüger, W. Dworschak, K. Jung, I. Mühling, F. Engelke, and H. Metz, Diamond Relat. Mater. 1, 316 (1992).

${ }^{20}$ R. Kleber, K. Jung, H. Ehrhardt, I. Mühling, K. Breuer, H. Metz, and F. Engelke, Thin Solid Films 205, 274 (1991).

${ }^{21}$ J. Schwan, S. Ulrich, H. Roth, K. Jung, H. Ehrhardt, R. Samlenski, R. Brenn, and S. R. P. Silva, J. Appl. Phys. 79, 1416 (1996).

${ }^{22}$ J. Schwan, S. Ulrich, K. Jung, H. Ehrhardt, R. Samlenski, and R. Brenn, Diamond Relat. Mater. 4, 304 (1995).

${ }^{23}$ W. S. Bacsa, J. S. Lannin, D. L. Pappas, and J. J. Cuomo, Phys. Rev. B 47, 10931 (1993).

${ }^{24}$ R. B. Wright, R. Varma, and D. M. Gruen, J. Nucl. Mater. 63, 415 (1976).

${ }^{25}$ W. Yarbrough and R. Rey, in Diamond and Related Materials, edited by A. Badzian, M. Geis, and G. Johnson (Materials Resource Society, Pittsburgh, PA, 1988), extended abstract Vol. EA-15, p. 33.

${ }^{26}$ S. R. P. Silva, G. Amaratunga, E. Salje, and K. Knowles, J. Mater. Res. 29, 4962 (1994).

${ }^{27}$ J. Kohanoff, Comput. Mater. Sci. 2, 221 (1994).

${ }^{28}$ D. Knight and W. White, J. Mater. Res. 4, 385 (1989).

${ }^{29}$ N. Colthup, L. Daly, and S. Wiberley, Introduction to Infrared and Raman Spectroscopy (Academic, New York 1975).
${ }^{30}$ M. Tamor, J. Haire, C. Wu, and K. Hass, Appl. Phys. Lett. 54, 123 (1989).

${ }^{31}$ M. Tamor, C. Wu, R. Carter, and N. Lindsay, Appl. Phys. Lett. 55, 1388 (1989).

${ }^{32}$ Sadtler Research Laboratories, Standard Raman Spectra, Vol. 9, 3555R, 3316 Spring Garden Street, Philadelphia, PA 19104.

${ }^{33}$ F. R. Dollish, W. G. Fateley, and F. F. Bentley, Characteristic Raman Frequencies of Organic Compounds (Wiley, New York 1974).

${ }^{34}$ H. Sakata, G. Dresselhaus, M. S. Dresselhaus, and M. Endo, J. Appl. Phys. 63, 2769 (1988).

${ }^{35}$ S. R. P. Silva, B. Rafferty, G. A. J. Amaratunga, L. M. Brown, J. Schwan, and D. Franceschini, Diamond Relat. Mater. 5, 401 (1996).

${ }^{36}$ G. Mariotto, F. Freire, and C. Achete, Thin Solid Films 241, 255 (1994).

${ }^{37}$ S. Prawer, K. W. Nugent, Y. Lifshitz, G. D. Lempert, E. Grossmann, J. Kulik, I. Avigal, and R. Kalish, Diamond Relat. Mater. (in press).

${ }^{38}$ J. Wagner, M. Ramsteiner, C. Wild, and P. Koidl, Phys. Rev. B 40, 1817 (1989).

${ }^{39}$ S. R. P. Silva, B. Rafferty, G. A. J. Amaratunga, J. Robertson, L. M. Brown, J. Schwan, G. Mariotto, and D. Franceschini (unpublished).

${ }^{40}$ C. A. Davis, S. R. P. Silva, K. M. Knowles, G. A. J. Amaratunga, and M. W. Stobbs, Phys. Rev. Lett. 75, 4258 (1995).

${ }^{41}$ F. Li and J. Lannin, Phys. Rev. Lett. 65, 1905 (1990).

${ }^{42}$ J. Ager III, S. Anders, A. Anders, and I. Brown, Appl. Phys. Lett. 66, 3444 (1995).

${ }^{43}$ M. Ramsteiner, J. Wagner, C. Wild, and P. Koidl, J. Appl. Phys. 62, 729 (1987). 